\title{
Superfluid Quantum Space as the Unified Field Theory
}

\author{
Amrit S. Šorli \\ Bijective Physics Institute, Slovenia \\ sorli.bijective.physics@gmail.com \\ https://orcid.org/0000-0001-6711-4844
}

\begin{abstract}
The novelty of 21st-century physics is the development of the "superfluid quantum vacuum" model, also named "superfluid quantum space" SQS. This SQS is replacing space-time as the fundamental arena of the universe. It also represents the model that has the potential of unifying four fundamental forces of the universe. SQS in this article is represented as the time-invariant fundamental field of the universe where time is merely the duration of material changes.
\end{abstract}

Keywords: unified field model, superfluid quantum space (SQS), gravity, entanglement, time

\section{Introduction}

Valeriy Sbitnev suggests that superfluid quantum vacuum also named superfluid quantum space (SQS) is the physical origin of the universal space [1,2]. In this article I developed a model of the time-invariant $n$-dimensional complex universal space which offers the new solution for Einstein's dream of a "Unified Field Model”. In Einstein's Relativity the universal space is understood as a 4-D reality with tree spatial dimensions and one temporal dimension. Bezuglav also suggested that the superfluid quantum vacuum, which is the physical origin of the universal space, is four-dimensional [3]. In experimental physics, time is duration of material change, i.e. motion in space. Taking this in account I developed the model of timeinvariant $\mathrm{n}$-dimensional complex universal space.

The measured value of cosmological constant $\Lambda=5.96 \cdot 10^{-27} \mathrm{~kg} / \mathrm{m}^{3}$ [4] is different from its calculated value following the Planck metrics for the magnitude of $10^{123}$; this discrepancy is an unsolved subject of physics for decades [5]. Regarding the suggested energy density of space proposed in this article, I'm defending my proposal by the fact that the gravitational constant $G$ is obtained by measurement and is expressed by the Planck energy density $\rho_{E P}$ and the Planck time $t_{\mathrm{P}}$ as [6]:

$$
G=\frac{c^{2}}{\rho_{E P} t_{P}^{2}}
$$


This means that the Planck energy density $\rho_{E P}$ reflects the real energy density of a 4-D universal space. In the absence of stellar objects, the energy density of the universal space has a value of Planck energy density which is $\rho_{E P}=4.64 \cdot 10^{113} \mathrm{Jm}^{-3}[6]$.

Meis has developed another formula for calculating the gravitational constant $G$ :

$$
G=\frac{l_{P}^{2} c^{2}}{4 \pi e \xi}
$$

where $e$ is the elementary charge constant and $\xi$ is the vector potential amplitude of the electromagnetic field to a single photon state $\left(\xi=1.747 \cdot 10^{-25} \mathrm{~V} \mathrm{~m}^{-1} \mathrm{~s}^{2}\right)$ [7].

We can replace in Eq. (2) the term $c^{2}$ with the electric permittivity $\varepsilon_{0}$ and the magnetic permeability $\mu_{0}$ obtaining:

$$
G=\frac{l_{P}^{2}}{4 \pi e \xi \varepsilon_{0} \mu_{0}}
$$

Eq. (3) confirms that the 4-D SQS electromagnetic properties are defining the gravitational constant.

\section{SQS as the unified field model}

Superfluid quantum space(SQS) has a general $n$-dimensional complex structure $\mathbb{C}^{\mathrm{n}}$; every point of it has complex coordinates:

$$
z_{i}=x_{i}+i y_{i}
$$

$\left(x_{i}, y_{i}\right)(i=1, \ldots, n)$ is an ordered $n$-uple of real numbers $\left(\left(x_{i}, y_{i}\right) \in \mathbb{R}^{\mathrm{n}}\right)$; for the purpose of this paper we consider its subset $\mathbb{C}^{4}$ where all elementary particles are different structures of $\mathbb{C}^{4-}$ SQS and have four complex dimensions $z_{i}$. In $\mathbb{C}^{\mathrm{n}}$-SQS the elapsed time of a given material change, i.e. motion is the sum of Planck times $t_{P}$ :

$$
t=t_{P 1}+t_{P 2}+\ldots+t_{P N}=\sum_{i=1}^{N} t_{P i} \quad \text { (5) [8]. }
$$

$\mathbb{C}^{\mathrm{n}}$-SQS is time-invariant in the sense that time is not its fourth dimension. Material changes run in time-invariant $\mathbb{C}^{\mathrm{n}}$-SQS and time is their duration. We do not have any experimental data that time is the fourth dimension of space and I suggest in this article a novel model where time is only the duration of change in time-invariant complex space. $\mathbb{C}^{\mathrm{n}}-\mathrm{SQS}$ is the physical origin of the universal space; I call it "four-dimensional complex superfluid quantum space" $\left(\mathbb{C}^{4}\right.$ SQS). Subatomic particles are different structures of $\mathbb{C}^{4}$-SQS; atoms, made out of subatomic particles, are three-dimensional physical objects, described by real geometry $\mathbb{R}^{3}$ and therefore 
follow the 3-D Euclidean geometry. Because of that we cannot fully grasp the complex subatomic level with 3-D apparatuses (Figure 1).

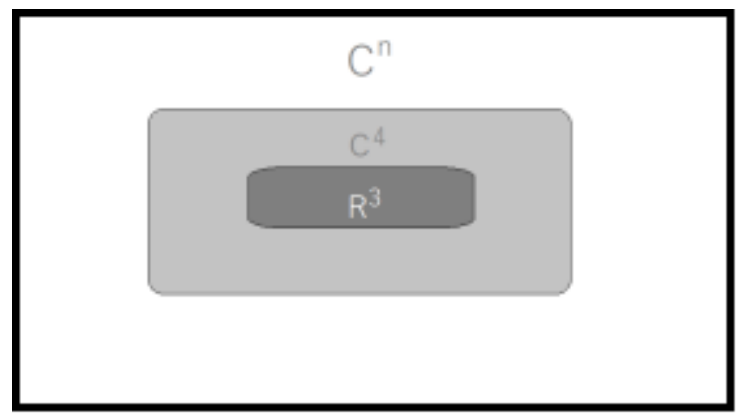

Figure 1: Structure of the $\mathbb{C}^{\mathrm{n}}-\mathrm{SQS}$ universe.

The 4-D complex superfluid quantum space $\mathbb{C}^{4}$-SQS is the theoretical frame for the unification of gravity and the other three fundamental forces which have already been unified by the Standard Model. In this complex superfluid quantum space, we have four spatial coordinates which have a real and imaginary component. The energy density of $\mathbb{C}^{4}$-SQS is calculated in the terms of $\mathbb{R}^{3}$ matter in units $\mathrm{kg} / \mathrm{m}^{3}$ and related to the mass $m$ of a given physical object; every physical object with mass $m$ is decreasing the energy density $\rho_{E \min }$ of $\mathbb{C}^{4}$-SQS in its centre exactly for the amount of its energy:

$$
E=m c^{2}=\left(\rho_{E P}-\rho_{E \min }\right) V
$$

where $\rho_{E P}$ is the energy density of SQS far away of a stellar object in the interstellar space and $V$ is its volume [6]. By Eq. (6) we can calculate the minimal energy density of space in the centre of a given physical object:

$$
\rho_{E \min }=\rho_{E P}-\frac{m c^{2}}{V}
$$

Eq. (7) holds from the proton scale to black holes scale. Going away from the centre of a given physical object, the energy density of space is increasing by the following equation [9]:

$$
\rho_{E \min }=\rho_{E P}-\frac{3 m c^{2}}{4 \pi(r+d)^{3}}
$$

where $r$ is the radius of the physical object and $d$ is the distance from its centre. When $d$ tends to the infinite, $\rho_{E \min }$ tends to $\rho_{E P}$ (Figure 2). 


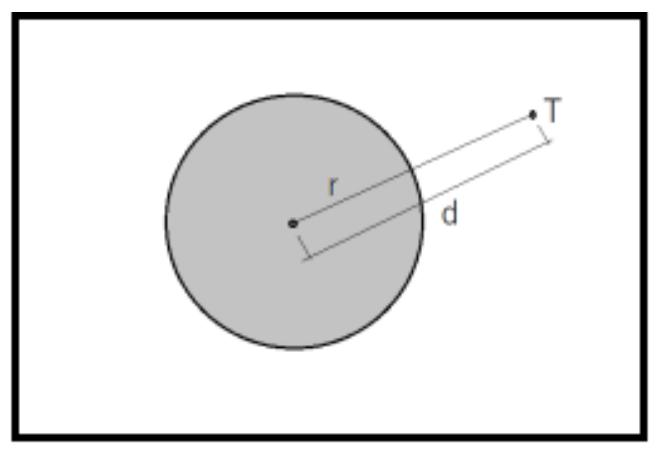

Figure 2. Energy density of $\mathbb{C}^{4}$-SQS in the point T at the distance $d$ from its centre.

Gravity is carried by external pressure of $\mathbb{C}^{4}$-SQS towards the centre of physical objects; it is the force of $\mathbb{C}^{4}$-SQS pressure from the maximum energy density of $\mathbb{C}^{4}$-SQS towards its decreased energy density in the centre of the given physical object. Two physical objects are creating decreased area of $\mathbb{C}^{4}$-SQS energy density, causing outer pressure of $\mathbb{C}^{4}$-SQS towards its lower inner pressure. This outer pressure is gravity (Figure 3) [6].

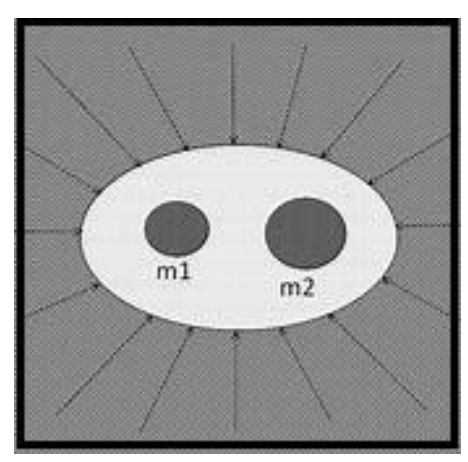

Figure 3. Gravity is the space pressure towards the physical objects.

I will use this Eq. (7) to calculate the energy density of space in the centre of different stellar objects, considering that these objects are non-rotating. In Table 1 there is the comparation of the energy densities of space in the centre of the black hole with the mass of the Sun, in the centre of the proton, in the centre of the Moon, Earth, and Sun:

Table 1. Comparation values of the minimal energy density of space with respect to the centre of indicated objects.

\begin{tabular}{|c|c|}
\hline Centre of objects & $\rho_{P}=4.64 \cdot 10^{113} \mathrm{Jm}^{-3}$ \\
\hline Black hole with mass of the Sun & $\rho_{P}-1.58 \cdot 10^{36} \mathrm{Jm}^{-3}$ \\
\hline
\end{tabular}




\begin{tabular}{|c|c|}
\hline Proton & $\rho_{P}-5.43 \cdot 10^{34} \mathrm{Jm}^{-3}$ \\
\hline Earth & $\rho_{P}-4.94 \cdot 10^{20} \mathrm{Jm}^{-3}$ \\
\hline Moon & $\rho_{P}-3.00 \cdot 10^{20} \mathrm{Jm}^{-3}$ \\
\hline Sun & $\rho_{P}-1.26 \cdot 10^{20} \mathrm{Jm}^{-3}$ \\
\hline
\end{tabular}

The energy density of space in the proton centre is lower than in the centre of Sun, Earth and Moon because these stellar objects are made out of atoms where there is a vast empty space between the nucleus and electrons orbits. Proton's mass is very small compared with the mass of the Sun, but it diminishes the energy density of an extremely small area of space compared with that of Sun, that diminishes the energy density of an extremely big area of universal space; that's why the gravity force of the Sun has such a long-range.

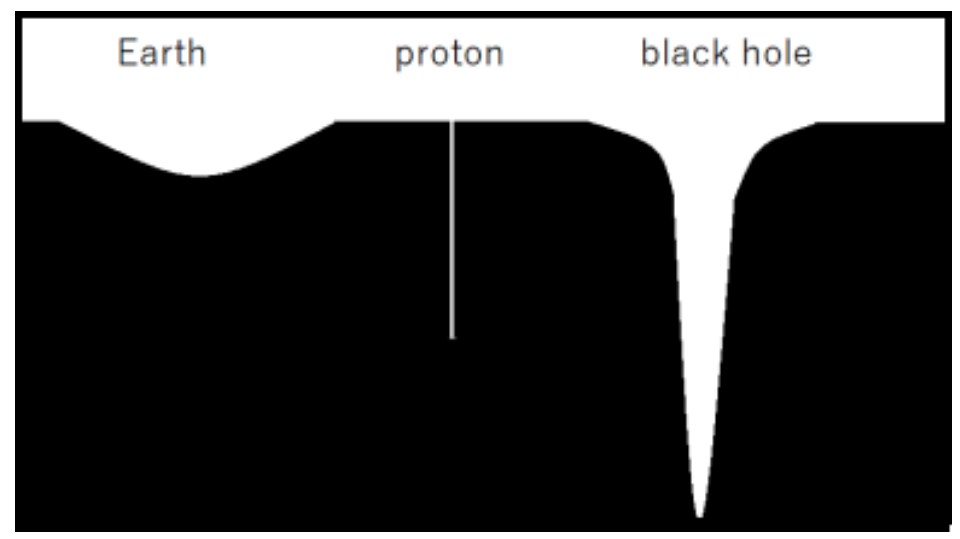

Figure 4: Energy density of $\mathbb{C}^{4}$-SQS in the centre of Earth, proton and black hole.

Proton has much lower energy density of $\mathbb{C}^{4}$-SQS in its centre than the Earth (Figure 4); however, it has almost no attraction force. The calculation of attraction force because of lower energy density of $\mathbb{C}^{4}$-SQS in the centre of proton and neutron in deuterium nucleus is as follows:

$$
F_{g}=\frac{1.67 \cdot 10^{-27} \mathrm{~kg} \cdot 1.67 \cdot 10^{-27} \mathrm{~kg} \cdot 6.67 \cdot 10^{-11} \mathrm{~m}^{3} \mathrm{~kg}^{-1} \mathrm{~s}^{-2}}{\left(2 \cdot 0.87 \cdot 10^{-15} \mathrm{~m}\right)^{2}}=6.026 \cdot 10^{-95} \mathrm{~N}
$$

where $1.67 \cdot 10^{-27} \mathrm{~kg}$ is the mass of proton and neutron, and $0.87 \cdot 10^{-15} \mathrm{~m}$ is their radius. We see that the energy density of $\mathbb{C}^{4}$-SQS in the proton centre is higher for the rate of $10^{2}$ than the correspondent energy density in the centre of the black hole. This shows that proton cannot 
be a mini black hole as suggested by Stephen Hawking [10]; Voyager did not find these primordial black holes suggested by him [11].

From the macro to the microscale it holds that a given physical object is interacting with the $\mathbb{C}^{4}$-SQS in which it exists and the result of this interaction are the inertial mass $m_{i}$ and the gravitational mass $m_{g}$ :

$$
m_{1}=m_{g}=\frac{\left(\rho_{E P}-\rho_{E \min }\right) V}{c^{2}}
$$

The rest mass $m_{0}$ of the proton is not its inertial mass $m_{i}$, but is related to the amount of $\mathbb{C}^{4}$-SQS energy $E$ which is incorporated in the proton, as per Eq. (9):

$$
\frac{E}{c^{2}}=m_{0}
$$

The inertial mass $m_{i}$ of the proton is the result of proton interaction with the $\mathbb{C}^{4}$-SQS energy and the decrease in energy density of $\mathbb{C}^{4}$-SQS is exactly for the amount of its mass and volume as we can see in Eq. (8). The decreased energy density of $\mathbb{C}^{4}$-SQS is the physical origin of proton's inertial mass. Einstein has proved that inertial mass and gravitational mass of a given physical object are equal; I have shown that they have the same origin in the decreased energy density of $\mathbb{C}^{4}$-SQS accordingly to the Eg. (8).

In the model of $\mathbb{C}^{4}$-SQS the electric field $\boldsymbol{E}$ is the excitation of $\mathbb{C}^{4}$-SQS on the three real dimensions $X_{1}, X_{2}, X_{3}$, and the magnetic field $\boldsymbol{B}$ the excitation of $\mathbb{C}^{4}$-SQS on the tree real dimension $X_{2}, X_{3}, X_{4}$. Both fields have in common dimensions $X_{2}$ and $X_{3}$. The photon is then the excitation of $\mathbb{C}^{4}$-SQS on $X_{1}, X_{2}, X_{3}, X_{4}$ dimensions, it is a 4-D wave of $\mathbb{C}^{4}$-SQS; the light has a constant speed for all moving observers because it is a wave of $\mathbb{C}^{4}$-SQS. When the source of light is moving closer to the observer or moving away from the observer the frequency of light will respect the Doppler effect. The source of light and the moving observer are all moving in the $\mathbb{C}^{4}$-SQS. This model explains the physical meaning of the first postulate of Special Relativity, i.e. that the light has the same velocity for all observers; the light is a 4-D wave of $\mathbb{C}^{4}$-SQS in which the observer and the 3-D source of light are moving. The motion of the observer or the motion of light source creates the Doppler effect but the light speed remains unchanged.

In Special Relativity the photon is moving in a 4-D space of Minkowski, where time $t$ is the element of the forth dimension $\mathrm{X}_{4}=i c t$. With Fiscaletti we have shown that time is just the numerical sequential order of material changes, i.e. motion in $\mathbb{C}^{4}$-SQS. The photon is the wave of $\mathbb{C}^{4}$-SQS and does not move in some physical time. When we measure the numerical order of photon motion from the point $\mathrm{A}$ to the point $\mathrm{B}$ in $\mathbb{C}^{4}$-SQS, we get duration [8]. 
The strong nuclear force is carried by gluons which bind together quarks inside the proton and neutron. In the model here presented gluons are excitations of $\mathbb{C}^{4}$-SQS and they represent $99 \%$ of proton mass. Residual nuclear force between quarks is acting also outside protons and neutrons and hold them together. In this perspective, the proton mass can be seen as the excitation of the $\mathbb{C}^{4}$-SQS in the form of gluons. In Meis model the mass of electron and proton is expressed by the physical properties of the electromagnetic vacuum:

$$
\begin{aligned}
& m_{e}=2 \pi c e^{2}\left|\frac{\xi}{\mu_{B}}\right|=9.109 \cdot 10^{-31} \mathrm{~kg} \\
& M_{P}=2 \pi c e^{2}\left|\frac{\xi}{\mu_{B}}\right|=1.672 \cdot 10^{-27} \mathrm{~kg}
\end{aligned}
$$

The mass $m_{i}$ of any elementary particle $i$ can be expressed using Eq. (13):

$$
m_{i}=2 \pi c e^{2}\left|\frac{\xi}{\mu_{i}}\right|
$$

with $\left|\mu_{i}\right|=\mu_{B}$ for the electron and $\left|\mu_{i}\right|=\left(2 \alpha / n_{i}\right)$ for other particles, with $n_{i}$ an integer and $\alpha$ the fine structure constant [7].

In Meis model, as well in $\mathbb{C}^{4}$-SQS model, elementary particles are different energy structures of the $\mathbb{C}^{4}-\mathrm{SQS}$ energy. This is also the view of Erwin Schrödinger who used to say: "What we observe as material bodies and forces are nothing but shapes and variations in the structure of space" [12]. This is expressed in Einstein formula $E=m c^{2} ; E$ is the $\mathbb{C}^{4}$-SQS energy which is incorporated in a given physical object, $m$ is the mass of the object.

Relativistic particles are interacting with the $\mathbb{C}^{4}$-SQS and additionally integrating $\mathbb{C}^{4}$-SQS energy into its structure. Relativistic energy $E$ of a given accelerated particle is the sum of the rest energy $E_{0}$ and kinetic energy $E_{K}$ which is incorporated energy of $\mathbb{C}^{4}$-SQS due to the motion of the particle:

$$
E=E_{0}+E_{K}=\gamma m_{0} c^{2}=\left(\rho_{E P}-\rho_{E m i n R}\right) V \quad(14)[7],
$$

where $\gamma$ is Lorentz factor, $m_{0}$ is proton rest energy, $\rho_{P}$ is Planck energy density, $\rho_{E m i n R}$ is additionally diminished energy density of $\mathbb{C}^{4}$-SQS in the centre of the proton because the proton is additionally absorbing $\mathbb{C}^{4}$-SQS energy and so increasing its mass and energy, $V$ is the volume of the proton at rest.

The unification of electromagnetism and weak nuclear force into electroweak force was independently proposed by Sheldon Glashow, Abdus Salam and Steven Weinberg in the sixties of the last century; we introduced here a model where all four fundamental forces are 
carried by $\mathbb{C}^{4}$-SQS. Gravity force is carried by variable density of $\mathbb{C}^{4}$-SQS, strong nuclear force and electroweak force are carried by the excitation of $\mathbb{C}^{4}-S Q S$.

\section{Complex time-invariant $\mathbb{C}^{4}$-SQS is the medium of quantum entanglement}

In the complex $\mathbb{C}^{4}$-SQS time is not the fourth dimension. In the complex $\mathbb{C}^{4}$-SQS imaginary dimensions $i y_{1}, i y_{2}, i y_{3}, i y_{4}$ act as the "hidden variables" proposed by Albert Einstein. These hidden variables enabling $\mathbb{C}^{4}$-SQS as the medium of the immediate information transfer by EPR-type experiments [13].

As the entire material world is made out of elementary particles which are structures of $\mathbb{C}^{4}$-SQS, we can say that the entire universe is entangled via $\mathbb{C}^{4}$-SQS. In this perspective, time as duration of material change, i.e. motion in $\mathbb{C}^{4}$-SQS, can be seen as an emergent property of entangled universe. Moreva et al. came to the same conclusion, namely time is an emergent property of entanglement, starting their research from a different perspective [14].

Einstein has interpreted the time $t$ as the $4^{\text {th }}$ coordinate $X_{4}$ of a Minkowski manifold. He wrote: "If we replace $x, y, z, \sqrt{-1} c t$ by $x_{1}, x_{2}, x_{3}, x_{4}$, we also obtain the result that $d s^{2}=d x_{1}^{2}+$ $d x_{2}{ }^{2}+d x_{3}^{2}+d x_{4}^{2}$ is independent of the choice of the body of reference. We call the magnitude $d s$ the "distance" apart of two events or four-dimensional points. Thus, if we choose as time variable the imaginary variable $\sqrt{-1} c t$ instead of the real quantity $t$, we can regard the continuum space-time, in accordance with the special theory of relativity, as an "Euclidean" four-dimensional continuum, a result following by the consideration of the preceding section" [15]. In the above citation, Einstein suggestion that we can choose the time variable $t$ as the imaginary variable can be written as follows:

$$
t=\sqrt{-1} c t
$$

Eq. (15) is false because on the left side of the equation we have $t$ and on the left side we have $\sqrt{-1} c t$. Combining Eq. (6) with equation well know equation $X_{4}=i c t$ we get:

$$
X_{4}=i t c^{2} \sqrt{-1}
$$

Einstein did a mistake keeping and interpreting time as the dimension of a four-dimensional continuum. Physics is still today suffering this misinterpretation of time that is solved in this article: time is the duration of a given physical object motion in time-invariant space. 
Several authors are proposing that entanglement is induced by gravity $[16,17,18]$. On the other hand, there is a proposal that entanglement influences gravity: "To summarize, we have shown that entanglement can affect the gravitational field. This suggests that entanglement "has a weight". The perturbations in the gravitational field depend on the amount of entanglement and vanish for vanishing quantum correlations" [19]. I have shown in this article that gravity and entanglement are carried by the same medium which is SQS. In the model presented in this article gravity force between two physical objects does not induce entanglement and entanglement has no impact on gravity. Two entangled physical objects are entangled via SQS which variable energy density is also carrying gravity. Gravity does not influence entanglement and vice versa is also valid. In the article here presented gravity and entanglement are both induced by the superfluid quantum space that is time-invariant.

\section{Conclusions}

The unified field theory of Albert Einstein is one of the main goals of modern physics. This goal can be achieved by the development of complex $\mathbb{C}^{4}-\mathrm{SQS}$ as the fundamental arena of the universe. Elementary particles and consequently strong nuclear force and electroweak force forces are different structures of $\mathbb{C}^{4}$-SQS. Gravity does not require the existence of some hypothetical particle graviton. It is carried directly by the variable energy density of timeinvariant complex $\mathbb{C}^{4}$-SQS that is the medium of quantum entanglement EPR-type phenomena.

\section{References:}

1. Sbitnev, V.I. Hydrodynamics of the Physical Vacuum: II. Vorticity Dynamics. Found Phys 46, 1238-1252 (2016). https://doi.org/10.1007/s10701-015-9985-3.

2. Sbitnev, V.I. Hydrodynamics of the Physical Vacuum: I. Scalar Quantum Sector. Found Phys 46, 606-619 (2016). https://doi.org/10.1007/s10701-015-9980-8.

3. Bezuglov, M, False vacuum decay in quantum mechanics and four dimensional scalar field theory, EPJ Web of Conferences, 177, 09001 (2018).

https://doi.org/10.1051/epjconf/201817709001.

4. Planck Collaboration, Planck 2015 results. XIII. Cosmological parameters, Astronomy \& Astrophysics, 594, A13 (2016). arXiv:1502.01589, doi:10.1051/0004-6361/201525830.

5. Peebles, P.J.E., Open problems in cosmology, Nuclear Physics B - Proceedings Supplements, 138, 5-9 (2005).

6. Fiscaletti, D., \& Šorli, A.S., Quantum Relativity: Variable Energy Density of Quantum Vacuum as the Origin of Mass, Gravity and the Quantum Behaviour, Ukrainian Journal of Physics, 63(7), 623 (2018). https://doi.org/10.15407/ujpe63.7.623. 
7. Meis C., Primary Role of the Quantum Electromagnetic vacuum in Gravitation and Cosmology (2020). doi: 10.5772/intechopen.91157.

8. Fiscaletti, D., Šorli, A. Perspectives of the Numerical Order of Material Changes in Timeless Approaches in Physics. Foundations of Physics, 45, 105-133 (2015). https://doi.org/10.1007/s10701-014-9840-y.

9. Šorli, A.S. Mass-Energy Equivalence Extension onto a Superfluid Quantum Vacuum, Scientific Reports, 9, 11737 (2019). https://doi.org/10.1038/s41598-019-48018$\underline{2}$.

10. Hawking, S., Gravitationally Collapsed Objects of Very Low Mass, Monthly Notices of the Royal Astronomical Society, 152(1), 75-78 (1971).

https://doi.org/10.1093/mnras/152.1.75.

11. Boudaud, M. \& Cirelli, M. Voyager $1 \mathrm{e}^{ \pm}$Further Constrain Primordial Black Holes as Dark Matter, Physical Review Letters, 122, 041104 (2019). https://journals.aps.org/prl/abstract/10.1103/PhysRevLett.122.041104.

12. Schrödinger, E., Space-Time Structure, Cambridge: Cambridge University Press (1985).

13. Fiscaletti, D., Šorli, A.S., Searching for an adequate relation between time and entanglement, Quantum Studies: Mathematics and Foundations, 4, 357-374 (2017). https://link.springer.com/article/10.1007/s40509-017-0110-5.

14. Moreva, E., Brida, G., Gramegna, M., Giovannetti, V., Maccone, L., and Genovese, M., Time from quantum entanglement: An experimental illustration, Physical Review A, 89, 052122 (2014). https://doi.org/10.1103/PhysRevA.89.052122.

15. Einstein, A., Relativity: The Special and General Theory, Methuen \& Co Ltd, p.93 (1916).

16. Krisnanda, T., Tham, G.Y., Paternostro, M. et al. Observable quantum entanglement due to gravity. npj Quantum Inf 6, 12 (2020). https://doi.org/10.1038/s41534-020-0243-y

17. Bose, S. et al. Spin entanglement witness for quantum gravity. Phys. Rev. Lett. 119, 240401 (2017). https://doi.org/10.1103/PhysRevLett.119.240401

18. Marletto, C. \& Vedral, V. Gravitationally induced entanglement between two massive particles is sufficient evidence of quantum effects in gravity. Phys. Rev. Lett. 119, 240402 (2017). https://doi.org/10.1103/PhysRevLett.119.240402

19. David Edward Bruschi, On the weight of entanglement, Physics Letters B, Volume 754, 10 March 2016, Pages 182-186, https://doi.org/10.1016/j.physletb.2016.01.034 